Research Article

Genomics and Bioinformatics

\title{
Identification of LincRNA from Dermatophagoides farinae (Acari: Pyroglyphidae) for Potential Allergen-Related Targets
}

\author{
Ying Zhou ${ }^{1}$, Meili Wu ${ }^{1}$, Hanting $\mathrm{Zhu}^{2}$, Junjie Shao ${ }^{3}$, Chang $\mathrm{Liu}^{3}$ and Yubao Cui ${ }^{2}$ (iE \\ ${ }^{1}$ Department of Pediatrics Laboratory, Wuxi Children's Hospital, Wuxi, China. \\ ${ }^{2}$ Department of Clinical Laboratory, Wuxi People's Hospital Affiliated to Nanjing Medical University, Wuxi, \\ China. \\ ${ }^{3}$ Chinese Academy of Medical Science, Institute of Medicinal Plant Development, Beijing, China.
}

\begin{abstract}
Long noncoding RNAs (IncRNAs), especially their important subclass of long intergenic noncoding RNAs (lincRNAs), have been identified in some insects. They play important roles in the regulation of biological processes, such as immune response or cell differentiation and as possible evolutionary precursors for protein coding genes. House dust mites (HDMs) are recognized as allergenic mites because allergens are found in their feces and bodies. Dermatophagoides farinae is one of the most important pyroglyphid mites because of its abundance in the household. To determine if lincRNAs can regulate allergen presentation in HDMs, we analyzed RNA-seq data for HDMs. We identified 11 lincRNAs that are related to mRNAs coding for allergens in HDMs. Using qRT-PCR, we amplified 10 lincRNAs and their putative target allergen-encoding mRNAs, confirming expression of these lincRNAs and allergen genes. The results suggest that lincRNAs might be involved in the regulation of allergen production in HDMs and might represent potential acaricidal candidates to inhibit mite allergen production.
\end{abstract}

Keywords: House dust mites (HDMs), Dermatophagoides farinae, long noncoding RNAs (lncRNAs), allergen, RNA-seq.

Received: July 26, 2019; Accepted: December 01, 2019.

\section{Introduction}

House dust mites (HDMs) are one of the most important worldwide causes of allergic diseases, including allergic asthma, allergic rhinitis, and atopic dermatitis. Common species of HDM, like Dermatophagoides pteronyssinus, Dermatophagoides farinae (D. farinae), and Blomia tropicalis (Calderon et al., 2015), produce allergenic proteins, i.e., that bind $\operatorname{IgE}$ from sera of $>5 \%$ of patients with symptoms of HDM allergies (Caraballo 2017; Thomas, 2018). To date, 39 groups of mite allergens have been reported. The World Health Organization and International Union of Immunological Societies (WHO/IUIS) subcommittee on allergen nomenclature has assigned official names for isolated allergens, which are listed at www.allergen.org. HDM allergens are present in mite bodies, secreta, and excreta, with feces being the major source of allergens.

Production of allergenic proteins is likely regulated by both transcriptional and post-translational processes. One potential source of regulation is long noncoding RNA (lncRNA), a class of abundant noncoding RNA that over-

Send correspondence to Yubao Cui, Department of Clinical Laboratory, Wuxi People's Hospital Affiliated to Nanjing Medical University, No. 299 at Qingyang Road, Wuxi 214023, Jiangsu Province, P. R. China. Email: ybcui1975@ hotmail.com. laps traditional coding genes and includes not only antisense, intronic, and intergenic transcripts but also pseudogenes and retrotransposons. IncRNAs are involved in various processes, such as cis and/or trans regulation of transcription, dosage compensation, imprinting, and competition with other endogenous RNAs (Li et al., 2014; Wang et al., 2018).

A limited number of insect genes has been experimentally annotated as lncRNAs. A 2012 study identified long non-coding intergenic RNAs (lincRNAs, a subset of lncRNAs transcribed from intergenic DNA) in insects using RNA-seq data, reporting 1,119 candidate lincRNA loci in the Drosophila melanogaster genome (Young et al., 2012). Some lncRNAs in Plutella xylostella, a pest of cruciferous plants, have been investigated in the context of insecticide resistance and might be involved in detoxification processes (Etebari et al., 2015). In addition, a relatively high-depth screen of 35 publicly available RNA-seq datasets from Aedes aegypti discovered 3,482 putative lincRNAs (Etebari et al., 2016). Finally, comparison of 14,161 lncRNAs from seven different species, including 1,559 from Tribolium castaneum, 2,602 from Drosophila melanogaster, 2,066 from Anopheles gambiae, 1,529 from Apis mellifera, 2,459 from Apis cerana, 2,176 from $\mathrm{Na}$ sonia vitripennis, and 1,770 from Drosophila pseudoobs- 
cura, showed low sequence conservation among different species. Further, similarities within a species are due to lncRNA association with transposable elements (TEs) and simple repeats, and TEs are less frequent in lincRNA exons than in introns, indicating that TEs may have been removed by selection (Lopez-Ezquerra et al., 2017).

To our knowledge, no lncRNAs have been explored in HDMs. Determining if HDMs contain lncRNAs will contribute to the understanding of allergen expression and regulation in HDMs. Previously, we used RNA-seq to identify HDM genes (Lin et al., 2018). Here, we identified $D$. farinae lncRNAs from RNA-seq data and confirmed their expression by qRT-PCR. Results of the current research will facilitate future studies to unravel the function of lncRNAs in HDMs and may help develop new methods to control HDMs or new immunotherapeutic strategies to treat allergies to mites.

\section{Materials and Methods}

\section{Mite culture and transcriptomic analysis}

$D$. farinae mites were isolated and cultured, and a cDNA library was constructed and sequenced according to previously reported methods (Lin et al., 2018). Briefly, total RNA extracted from $\sim 2,000$ homogenized mites was used to construct a transcriptome library with a SMART cDNA Library Construction Kit (American Clontech Corporation, \#634901). Sequencing was performed using an Illumina HiSeq 2500 sequencer, followed by de novo assembly with the Trinity/Oases suite.

\section{Total lincRNA and allergen-related lincRNA identification}

LincRNAs and their targeted mRNAs were identified from RNA-seq data using a computational pipeline ( $\mathrm{Li}$ et $a l ., 2014)$. To identify lincRNAs and mRNAs related to potential allergens, the sequences of all genes encoding known allergens were downloaded from www.allergen.org, the official site for systematic allergen nomenclature. Sequences were then searched against mRNAs and lincRNAs using BLASTN with a cutoff e-value of 1e-5. A1lergen sequences were selected and clustered.

\section{Validation of lincRNA from $D$. farinae}

Strand-specific real-time RT-PCR was used to quantify lincRNAs and target mRNAs coding for mite allergens. In brief, the miRNeasy Micro Kit (Qiagen, \#217084) was used to isolate total RNA (TaKaRa Biotech, Dalian, China, \#D312), and the PrimeScript ${ }^{\mathrm{TM}}$ RT reagent kit (TaKaRa, \#RR037A) was used for reverse transcription. The reverse transcription reaction system included $3 \mu \mathrm{L}$ of total RNA (1,000 ng), $2 \mu \mathrm{L}$ of $5 \mathrm{X}$ PrimeScript Buffer, $0.5 \mu \mathrm{L}$ of RT primer (final concentration $100 \mathrm{nM}$ ), $0.5 \mu \mathrm{L}$ of PrimeScript RT Enzyme Mix I, and DEPC-treated $\mathrm{ddH}_{2} \mathrm{O}$ to a final volume of $10 \mu \mathrm{L}$. Reverse transcription was carried out in an ABI 9700 PCR thermocycler at $42{ }^{\circ} \mathrm{C}$ for $15 \mathrm{~min}, 85^{\circ} \mathrm{C}$ for
$5 \mathrm{~s}$, and then held at $4{ }^{\circ} \mathrm{C}$. A SYBR Green PCR kit (TaKaRa, \#RR820A) was used for real-time PCR, performed in a total volume of $20 \mu \mathrm{L}$ containing $10 \mu \mathrm{L}$ of $2 \mathrm{X}$ Real-time PCR Master Mix, $2 \mu \mathrm{L}$ F primer (final concentration $1 \mu \mathrm{M}$ ), $2 \mu \mathrm{L}$ R Primer (final concentration $1 \mu \mathrm{M}$ ), 2

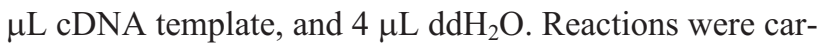
ried out on the LightCycler 480 real-time PCR instrument (Roche) at $95^{\circ} \mathrm{C}$ for $30 \mathrm{~s}$, followed by 40 cycles at $95^{\circ} \mathrm{C}$ for $10 \mathrm{~s}$ and $60^{\circ} \mathrm{C}$ for $50 \mathrm{~s}$. cDNA was denatured at $95^{\circ} \mathrm{C}$, followed by 40 cycles of $95^{\circ} \mathrm{C}$ for $10 \mathrm{~s}$ and $60{ }^{\circ} \mathrm{C}$ for $50 \mathrm{~s}$. Melting curve analysis was performed to validate generation of expected PCR products. The setting was $65^{\circ} \mathrm{C}$ to $95^{\circ} \mathrm{C}$ at a rate of $0.2^{\circ} \mathrm{C}$ per $10 \mathrm{~s}$. Product was detected by incubation at $95^{\circ} \mathrm{C}$ for $5 \mathrm{~s}, 65^{\circ} \mathrm{C}$ for $15 \mathrm{~s}$, and then held at $95{ }^{\circ} \mathrm{C}$. Primer sequences for real-time RT-PCR are shown in Table 1. Data for real-time RT-PCR were expressed using the $2^{-\Delta \Delta C t}$ method and analyzed in Excel.

\section{Results}

\section{Computational identification of $D$. farinae lincRNA}

We used a bioinformatics pipeline to identify lincRNAs from a set of strand-nonspecific RNA-seq data generated for $D$. farinae. From a total of 186,273 transcripts, 126,664 lincRNAs were predicted. We searched all known allergenic $D$. farinae proteins to find related lincRNAs. Identified lincRNAs and mRNAs were used to search against known allergenic $D$. farinae proteins. A total of 11 lincRNAs and 11 mRNAs related to mite allergens were expressed, some with levels indicating possible coordination (Table 2). These allergens were heat shock protein 70, ferritin, Der f 3, Der f 15, Der f 16, Der f 20, Der f 24, Der f 26, Der f 30, Der f 31, and Der f 33. Sequences for the 11 related lincRNAs are shown in Data $\mathrm{S} 1$.

\section{Strand-specific real-time RT-PCR identification of lincRNAs and mRNAs related to potential $D$. farinae allergens}

Strand-specific real-time RT-PCR was used to amplify the 11 lincRNAs and their associated mRNAs from $D$. farinae total RNA. Although one lincRNA (TRINITY_DN55999) failed to generate a curve, the other 10 lincRNAs and their putative target allergen-encoding mRNAs successfully amplified (Figure 1). Low levels of Der f 3 and Der f 20 mRNA were measured in the presence of higher levels of their corresponding lincRNAs, TRINITY_DN39419 and TRINITY_DN55962, indicating a possible negative interaction. However, mRNA expression levels of Der f 15, Der f 16, Der f24, Der f26, Der f 30, Der f 31, Der f 33, and heat shock protein 70 positively associated with expression of their corresponding lincRNAs. 
Table 1 - Oligonucleotide sets for strand-specific real-time RT-PCR using tagged primers to quantify lncRNA and targeted mRNA encoding mite allergens

\begin{tabular}{|c|c|c|}
\hline Gene symbol or GenBank accession number & Primer name & Primer sequence* \\
\hline \multirow[t]{3}{*}{ TRINITY_DN46653 } & $11-\mathrm{RT}$ & GGCCGTCATGGTGGCGAATCATCGGAAACTATATCGAATGATTC \\
\hline & $11-\mathrm{R}$ & GGCCGTCATGGTGGCGAAT \\
\hline & $11-\mathrm{F}$ & ATCAGTGTTTTACCTTCCGTAT \\
\hline \multirow[t]{3}{*}{ KM010005.1 } & $12-\mathrm{RT}$ & GCTAGCTTCAGCTAGGCATCCCATTACGTGTATTACATTTAACC \\
\hline & $12-\mathrm{R}$ & GCTAGCTTCAGCTAGGCATC \\
\hline & $12-\mathrm{F}$ & CGTCAATTACGGCATCATTA \\
\hline \multirow[t]{2}{*}{ TRINITY_DN13286 } & $21-\mathrm{F}$ & TCTGCCCTCGATATTCTGA \\
\hline & $21-\mathrm{R}$ & TTGATTCCTTGGATATTGCAC \\
\hline \multirow[t]{2}{*}{ KM009996.1 } & $22-\mathrm{F}$ & ATGGCTGATTTAAGACCAC \\
\hline & $22-\mathrm{R}$ & CTTTATCTTTCTTTACTTGGCTA \\
\hline \multirow[t]{3}{*}{ TRINITY_DN20632 } & $31-\mathrm{RT}$ & GGCCGTCATGGTGGCGAATTCATCAAGCGTAACACTACTGTCCC \\
\hline & $31-\mathrm{F} 1$ & GAAGGTAACTTCGATTTGTGG \\
\hline & $31-\mathrm{R}$ & GGCCGTCATGGTGGCGAAT \\
\hline \multirow[t]{3}{*}{ KC305502.1 } & $32-\mathrm{RT}$ & GCTAGCTTCAGCTAGGCATCGTTGGTCTTGCCAGTGCCCTTCTC \\
\hline & $32-\mathrm{F}$ & TGAGCGTGCTCGCACCAA \\
\hline & $32-\mathrm{R}$ & GCTAGCTTCAGCTAGGCATC \\
\hline \multirow[t]{2}{*}{ TRINITY_DN39419 } & $41-\mathrm{F}$ & TTATTGGCTGCAAACACTTG \\
\hline & $41-\mathrm{R}$ & TCTGATCCTTGTGGTGGC \\
\hline \multirow[t]{2}{*}{ D63858.1 } & $42-\mathrm{F}$ & TTATTGGCTGCAAACATTTT \\
\hline & $42-\mathrm{R}$ & TCTGATCCTTGTGCTGGT \\
\hline \multirow[t]{2}{*}{ TRINITY_DN51892 } & $51-\mathrm{F}$ & GCTCTATATATCATCGGTTGC \\
\hline & $51-\mathrm{R}$ & ATAAATAATAAAATGAAATG \\
\hline \multirow[t]{2}{*}{ AY283280.1 } & $52-\mathrm{F}$ & TCACAATCTAAAATGGCACT \\
\hline & $52-\mathrm{R}$ & ATAAAATTGGGTATGATACGAT \\
\hline \multirow[t]{3}{*}{ TRINITY_DN55962 } & $61-\mathrm{RT}$ & GGCCGTCATGGTGGCGAATGATCAACATTGACAAAGTGTTCG \\
\hline & $61-\mathrm{F}$ & AGATCAACTCAAGACGAAA \\
\hline & $61-\mathrm{R}$ & GGCCGTCATGGTGGCGAAT \\
\hline \multirow[t]{3}{*}{ KM009994.1 } & $62-\mathrm{RT}$ & GCTAGCTTCAGCTGAGCCGATCCACATTACACAAAGTGTTA \\
\hline & $62-\mathrm{R}$ & GCTAGCTTCAGCTGAGCC \\
\hline & $62-\mathrm{F}$ & GGATGCATGTAAAGGTCGT \\
\hline \multirow[t]{2}{*}{ TRINITY_DN43505 } & $71-\mathrm{F}$ & CTATATGAATAAGCGATCCAAC \\
\hline & $71-\mathrm{R}$ & TTTCCGACAATTAATTCGTTC \\
\hline \multirow[t]{2}{*}{ KC669700.1 } & $72-\mathrm{F}$ & TATCGTTTAGTTCGTGCAT \\
\hline & $72-\mathrm{R}$ & TATATTCAATAGTTCGCTCGT \\
\hline \multirow[t]{3}{*}{ TRINITY_DN55882 } & $81-\mathrm{RT}$ & GGCCGTCATGGTGGCGAATCAACGGGTTAATAAATTTGAT \\
\hline & $81-\mathrm{F}$ & CGGCCACTTTTATCCTCTT \\
\hline & $81-\mathrm{R}$ & GGCCGTCATGGTGGCGAAT \\
\hline \multirow[t]{3}{*}{ AF465625.1 } & $82-\mathrm{RT}$ & GCTAGCTTCAGCTAGGCATCTATCCTCTTCCAAATCATCT \\
\hline & $82-\mathrm{F}$ & GGAAGGTGATGAAAGTGTTG \\
\hline & $82-\mathrm{R}$ & GCTAGCTTCAGCTAGGCATC \\
\hline \multirow[t]{3}{*}{ TRINITY_DN54769 } & 91-RT & GGCCGTCATGGTGGCGAATTGTCGATGGACATCTTATCA \\
\hline & $91-\mathrm{F}$ & CATCGTTCGGTCTTTCGTT \\
\hline & $91-\mathrm{R}$ & GGCCGTCATGGTGGCGAAT \\
\hline \multirow[t]{3}{*}{ AF178772.1 } & 92-RT & GCTAGCTTCAGCTAGGCATCTTATTCGCCTATACAAGTCA \\
\hline & $92-\mathrm{F}$ & AACACCAGCCCCTACAACAT \\
\hline & $92-\mathrm{R}$ & GCTAGCTTCAGCTAGGCATC \\
\hline \multirow[t]{2}{*}{ TRINITY_DN8214 } & $101-\mathrm{F}$ & CACGTATGCAAAGATAGCAG \\
\hline & $101-\mathrm{R}$ & AAAGAGGCAAAAGATACAGA \\
\hline \multirow[t]{2}{*}{ KM010014.1 } & $102-\mathrm{F}$ & TATTGAAGTTGAAACTACTGGC \\
\hline & $102-\mathrm{R}$ & TTTATAAACACCGACAAGAGC \\
\hline TRINITY_DN55999 & $111-\mathrm{F}$ & AAAAAACTGTCAATCAAT \\
\hline & $111-\mathrm{R}$ & TCATCATCATTGTAGG \\
\hline КC305503.1 & $112-\mathrm{F}$ & AAAAAACAGTCAATCAGG \\
\hline & $112-\mathrm{R}$ & TTACGATGAATGCAAT \\
\hline
\end{tabular}

*RT primers contained six random bases. 


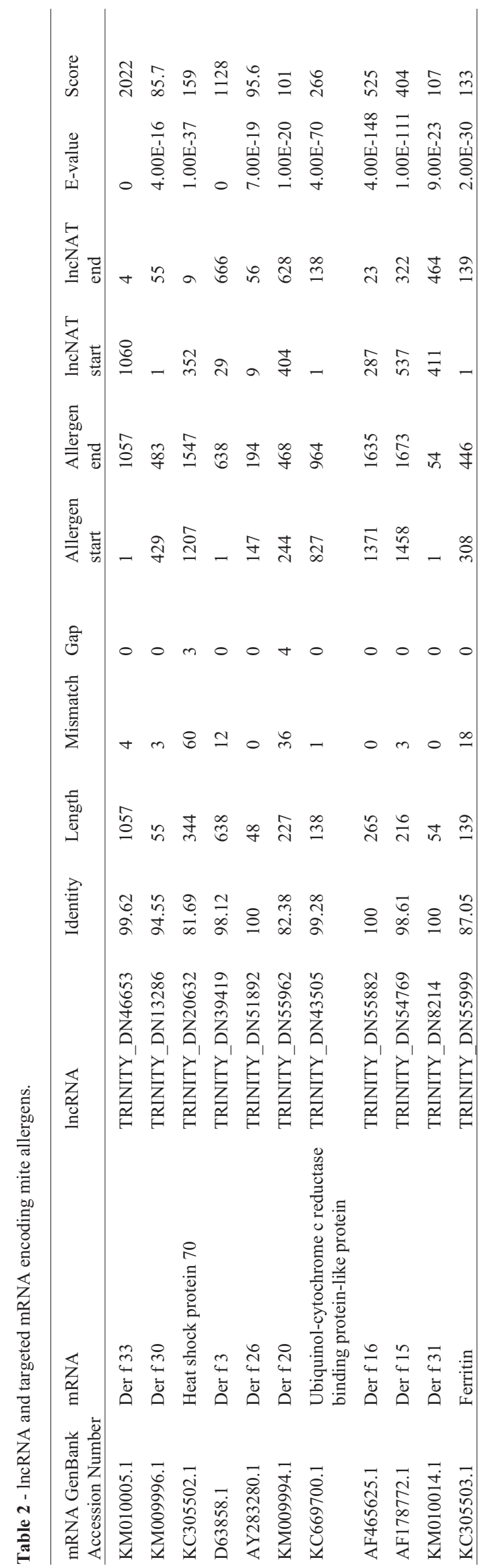

\section{Discussion}

The present study is the first to identify lincRNAs from HDMs and to explore their potential functions. We used our previously reported RNA-seq data from $D$. farinae (Peng et al., 2018) to generate a computational pipeline (Li et al., 2014) that identified 126,664 lincRNAs from 186,273 predicted transcripts. To determine how many of these lincRNAs are related to HDM allergens, we searched mRNA sequences coding for 37 groups of mite allergens using lincRNAs as query sequences. A total of 11 lincRNAs corresponding to $11 \mathrm{mRNAs}$ related to mite allergens were identified. Although the gene encoding ferritin and its lincRNA could not be amplified, strand-specific real-time RT-PCR identified the other 10 lincRNAs and their allergen-related potential target mRNAs in total mite RNA.

Real-time RT-PCR results showed that mRNA expression levels of Der $\mathrm{f} 3$ and Der $\mathrm{f} 20$ negatively correlated with expression of their corresponding lincRNAs, but mRNA expression levels of Der f 15, Der f 16, Der f24, Der f 26, Der f 30, Der f 31, Der f 33, and heat shock protein 70 positively associated with expression of their lincRNAs. According to a previous report, recombinant Der $\mathrm{f} 33$ reacts to the serum of patients with mite allergies, with a $23.5 \%$ positive rate for the skin prick test. In an asthma mouse model, Der f 33 induces airway allergy-like responses (Wang et al., 2016). Further, immunoblotting showed that $63.4 \%$ of dust mite allergic patients react to Der f 26 (An et al., 2013). Enzyme immunoassays indicated that recombinant Der f 16, prepared using an Escherichia coli expression system, binds IgE from 47\% (8/17) of mite-allergic patients (Kawamoto et al., 2002). In addition, 43 HDMallergic patients have shown $32.5 \%$ positive responses to skin prick tests for recombinant Der f 31 (Lin et al., 2018).

Aberrant expression of IncRNAs has been reported in immune-related diseases, including allergic diseases. For example, patients with eosinophilic esophagitis, an allergic inflammatory disorder, have altered lncRNA profiles (Sherrill et al., 2014), and altered lincRNA expression has been identified in CD8+ T cells of severe asthma patients (Tsitsiou et al., 2012). lincRNAs are involved in the innate immune response, including activation of monocytes and macrophages as well as regulating expression of inflammatory genes (Hadjicharalambous and Lindsay, 2019), highlighting potential pathways through which lincRNAs may mediate allergenic responses. However, few studies have examined lncRNAs with regards to HDM allergens. Our previous results show that exposing cells to HDM extracts results in differential expression of 270 lncRNAs, 119 of which were co-expressed with mRNAs. Bioinformatic analysis suggested these lncRNAs may target gene pathways related to glycolysis, axon guidance, ErbB signaling, and MAPK signaling (Wang et al., 2018).

In this study, we identified lncRNAs related to allergens produced by the important HDM D. farinae. One po- 

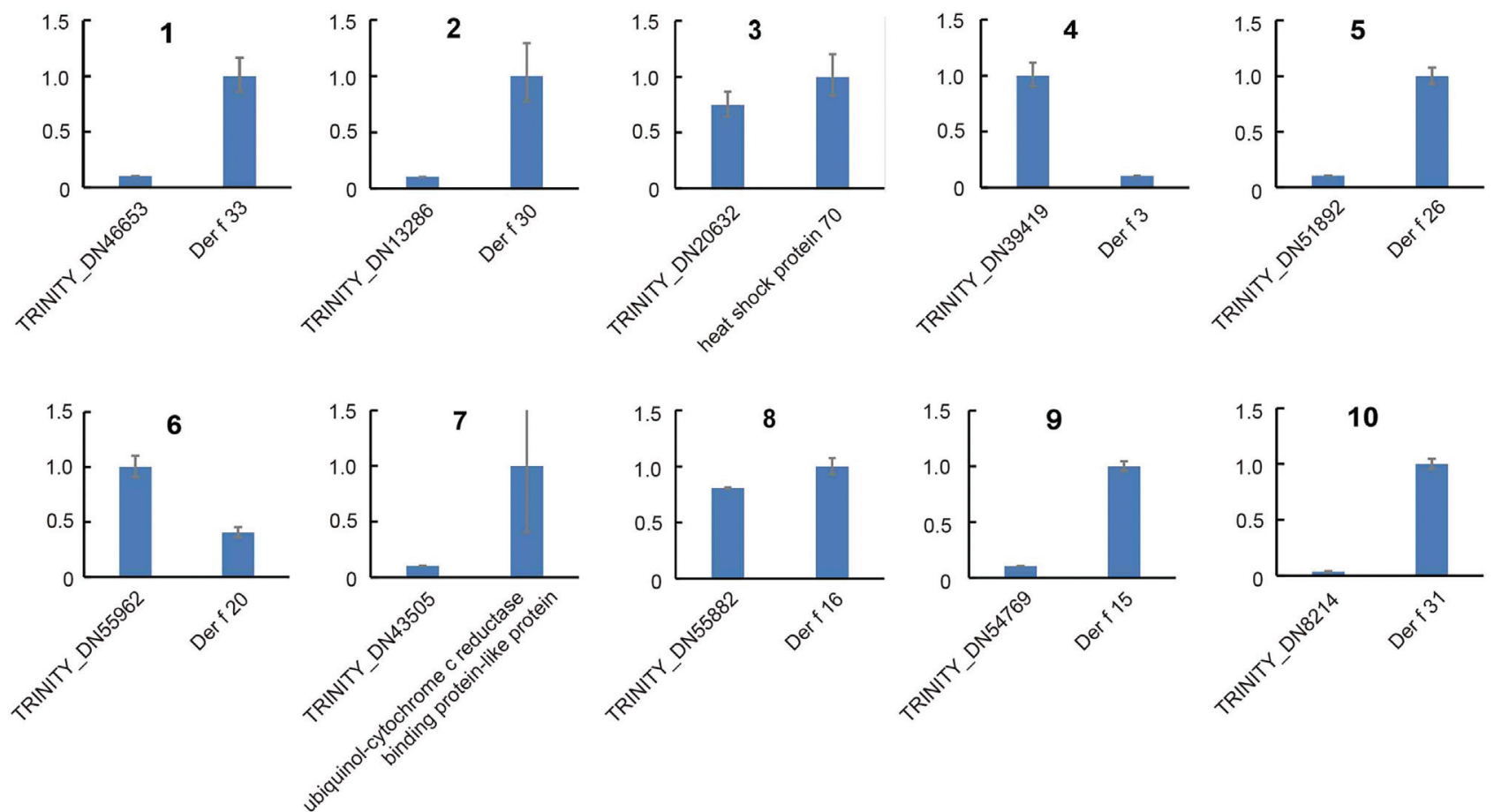

Figure 1 - qRT-PCR validation of expression of lincRNAs and mRNAs coding for allergens: (1) TRINITY DN46653 and Der $\mathrm{f} 33$; (2) TRINITY_DN13286 and Der f 30; (3) TRINITY_DN20632 and heat shock protein 70; (4) TRINITY_DN39419 and Der f 3; (5) TRINITY_DN51892 and Der f 26; (6) TRINITY_DN55962 and Der f 20; (7) TRINITY_DN43505 and Der f 24; (8) TRINITY_DN55882 and Der f 16; (9) TRINITY_DN54769 and Der f 15 ; and (10) TRINITY_DN8214 and Der f 31 .

tential application of lincRNAs is the development of acaricidal candidates to inhibit mite allergen production in homes. However, to test that possibility, we must first validate the functions of these lincRNAs by developing an asthmatic animal model sensitized by mRNA coding for allergens and then treat animals with corresponding lncRNAs. This is a logical next step in future research.

\section{Acknowledgments}

This study was supported by the National Natural Sciences Foundation of China (Grant No. NSFC31272369), the 333 project of Jiangsu Province in 2017 (BRA2017216), the Major Program of Wuxi health and Family Planning Commission (Z201701), and the Primary Research \& Development Plan of Jiangsu Province (Grant No.BE2018627).

\section{Conflicts of Interest}

The authors declare that there is no conflict of interest.

\section{Author contributions}

$\mathrm{CL}$ and $\mathrm{YC}$ conceived and designed the study, YZ, $\mathrm{MW}$, and $\mathrm{HZ}$ conducted the qRT-PCR. JS conducted the RNA-seq data analysis, $\mathrm{YC}$ and $\mathrm{YZ}$ contributed to writing the manuscript. All authors read and approved the final version of the manuscript.

\section{References}

An S, Chen L, Long C, Liu X, Xu X, Lu X, Rong M, Liu Z and Lai R (2013) Dermatophagoides farinae allergens diversity identification by proteomics. Mol Cell Proteomics 12:1818-1828.

Calderon MA, Linneberg A, Kleine-Tebbe J, De Blay F, Hernandez Fernandez de Rojas D, Virchow JC and Demoly P (2015) Respiratory allergy caused by house dust mites: What do we really know? J Allergy Clin Immunol 136:3848.

Caraballo L (2017) Mite allergens. Expert Rev Clin Immunol 13:297-299.

Etebari K, Asad S, Zhang G and Asgari S (2016) Identification of Aedes aegypti Long Intergenic Non-coding RNAs and Their Association with Wolbachia and Dengue Virus Infection. PLoS Negl Trop Dis 10:e0005069.

Etebari K, Furlong MJ and Asgari S (2015) Genome wide discovery of long intergenic non-coding RNAs in Diamondback moth (Plutella xylostella) and their expression in insecticide resistant strains. Sci Rep 5:14642.

Hadjicharalambous MR and Lindsay MA (2019) Long non-coding RNAs and the innate immune response. Noncoding RNA 5:34.

Kawamoto S, Suzuki T, Aki T, Katsutani T, Tsuboi S, Shigeta S and Ono K (2002) Der f 16: a novel gelsolin-related molecule identified as an allergen from the house dust mite, Dermatophagoides farinae. FEBS Lett 516:234-238.

Li J, Wu B, Xu J and Liu C (2014) Genome-wide identification and characterization of long intergenic non-coding RNAs in Ganoderma lucidum. PloS One 9:e99442. 
Lin J, Huang N, Wang H, Fu Q, Wang E, Li P, Yang L, Luo X, Liu $X$ and Liu Z (2018) Identification of a novel cofilin-related molecule (Der f 31 ) as an allergen from Dermatophagoides farinae. Immunobiology 223:246-251.

Lopez-Ezquerra A, Harrison MC and Bornberg-Bauer E (2017) Comparative analysis of lincRNA in insect species. BMC Evol Biol 17:155.

Peng J, Zhou Y, Jia H, Li L, Qian J, Han F, Yin H and Cui Y (2018) Transcriptomics-based identification of aquaporin diversity in the house dust mite Dermatophagoides farinae (Acariformes: Pyroglyphidae). J Insect Sci 18:11-9.

Sherrill JD, Kiran KC, Blanchard C, Stucke EM, Kemme KA, Collins MH, Abonia JP, Putnam PE, Mukkada VA, Kaul A et al. (2014) Analysis and expansion of the eosinophilic esophagitis transcriptome by RNA sequencing. Genes Immun 15:361-369.

Thomas WR (2018) Blueprint for the House Dust Mite. Int Arch Allergy Immunol 175:121-125.

Tsitsiou E, Williams AE, Moschos SA, Patel K, Rossios C, Jiang X, Adams OD, Macedo P, Booton R, Gibeon D et al. (2012) Transcriptome analysis shows activation of circulating CD8+ T cells in patients with severe asthma. J Allergy Clin Immunol 129:95-103.
Wang H, Lin J, Liu X, Liang Z, Yang P, Ran P and Liu Z (2016) Identification of alpha-tubulin, Der f 33, as a novel allergen from Dermatophagoides farinae. Immunobiology 221:911-917.

Wang L, Zhou Y and Cui Y (2018) lncRNA profiling in NCI-H292 cells after stimulation with Dermatophagoides farinae extracts. Int J Immunopathol Pharmacol 32:394632017750997.

Young RS, Marques AC, Tibbit C, Haerty W, Bassett AR, Liu JL and Ponting CP (2012) Identification and properties of 1,119 candidate lincRNA loci in the Drosophila melanogaster genome. Genome Biol Evol 4:427-442.

\section{Supplementary material}

The following online material is available for this article: Data Sl - Sequences of lincRNAs identified from this study.

Associate Editor: Marcela Ullano-Silva

License information: This is an open-access article distributed under the terms of the Creative Commons Attribution License (type CC-BY), which permits unrestricted use, distribution and reproduction in any medium, provided the original article is properly cited. 